\title{
Clinical Osteopathic Observation of Patients with Left- Ventricular Assistance Device
}

\section{Objective}

The objective of the study was to investigate the presence and location of osteopathic dysfunctions in this patient population, through palpation by qualified personnel such as qualified osteopaths D.O. Methods: The palpation evaluation concerned the spine, the respiratory diaphragm, the thoracic diaphragm, pelvic and buccal diaphragms. An evaluation of the craniosacral rhythm was also undertaken. Manual osteopathic evaluation was completed by the department on the second day after taking charge of the patient, before starting the cycle of cardiovascular rehabilitation. Results: We detected hypertonia of the paraspinal muscles at the level of C1-C2, C3-C4 and C7-T4, and highlighted that all patients have thoracic outlet syndrome. The dysfunction of the respiratory diaphragm reflects a dysfunction of the same side of the buccal and pelvic floors. Palpation of craniosacral rhythm showed a slowing of the cranial pulse. Conclusions: It is hoped that the information highlighted is used in choosing the type of therapy by manual practitioners, such as osteopaths, physical therapists and chiropractors, and that it will encourage other researchers to conduct further research in the manual field and patients with L- VAD.

Keywords: Osteopathic; Left ventricular assist device; Fascia; Diaphragm; Rehabilitation

\begin{tabular}{l} 
Clinical Paper \\
Volume 8 Issue 2 - 2017 \\
Bordoni B ${ }^{1 *}$, Marelli $\mathbf{F}^{2}$, Morabito B $^{2}$ and \\
Sacconi B ${ }^{3,4}$ \\
${ }^{1}$ Department of Cardiology, Institute of Hospitalization and \\
Care with Scientific Address, Italy \\
${ }^{2}$ CRESO, School of Osteopathic Centre for Research and Studies, \\
Italy \\
${ }^{3}$ Department of Radiological, Oncological and \\
Anatomopathological Sciences, Italy \\
${ }^{4}$ Center for Life Nano Science@Sapienza, Istituto Italiano di \\
Tecnologia, Italy \\
*Corresponding author: Bordoni Bruno, Foundation Don \\
Carlo Gnocchi IRCCS, Department of Cardiology, Institute \\
of Hospitalization and Care with Scientific Address, S \\
Maria Nascente, Via Capecelatro 66, Milan 20100, Italy, Tel: \\
0039.02.3496300617; Email: bordonibruno@ @otmail.com \\
Received: July 14, 2017 | Published: August 01, 2017 \\
\hline
\end{tabular}

Abbreviations: CHF: Chronic Heart Failure; EBM: EvidenceBased Medicine; L-VAD: Left Ventricular Assist Device

\section{Introduction}

Chronic heart failure (CHF) is a condition characterized by the inability of the heart to ensure proper supply of oxygen to the body tissues [1]. The prevalence of this disease is found mainly in patients over 50 years of age. 1 Heart disease from chronic insufficiency is increasing, due to the average increase in the age of the population and medical treatment that guarantees longer survival for the cardiac patient [1-3]. When heart failure is in an advanced stage and the clinical condition allows, the patient can be put on the transplant list. However, the number of organs available for transplant is lower than the demand $[4,5]$. Another treatment option in advanced heart failure is mechanical ventricular support, which can be offered to patients who meet pre-determined criteria for inclusion [6]. The most commonly surgically implanted device is left-ventricular assistance device. (L-VAD), a mechanical pump that supports the cardiac pump function, intervening in support of left ventricle in severe dysfunction, and which, in the newer models, provides a continual flow [7]. Connected to a pump; there is a cannula for the outflow that exits from the aorta, and one for the inflow which enters in the apex of the left ventricle. A package of external batteries for the operation of the pump is put into connection by means of an electric wire, called the drive line, which exits at the level of the subcutaneous tissue of the chest or peritoneum [7]. The system of external battery has a limited duration, approximately 12 hours in the newer models, and must be changed periodically during the day, put under electric charge. The patient must learn to manage the device and its management, thanks to the training provided by specialists. Since the system is carried out with the ultimate aim of having a transplant, it is referred to as "bridge therapy", while if the use of L-VAD becomes a definitive therapeutic solution, it is described as a "destination therapy" [5,8], (Figure 1).

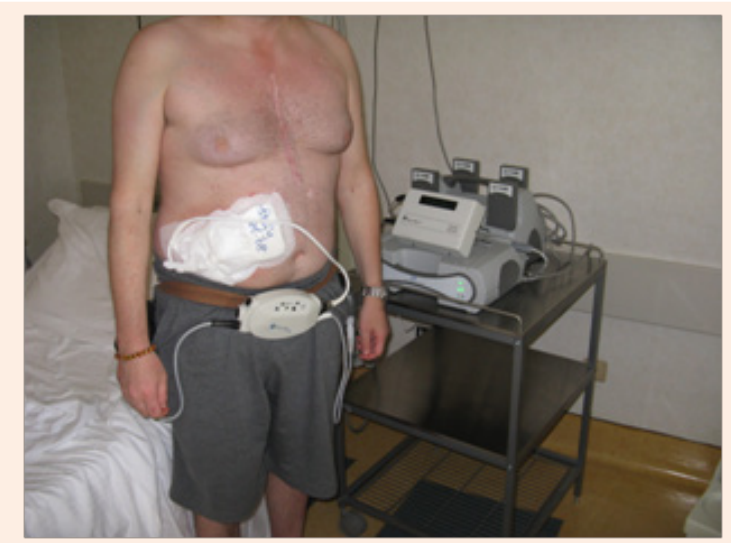

Figure 1: Patient with L-VAD.

To his left the machinery that carries replacement batteries, and instrumentation with a display where every day the medical staff evaluates the values of the patient, as the cycles per minute and the speed of the flow. The wire comes out of the peritoneum, in connection with the main control unit or controller (front) and the batteries on either side of the patient with a special belt. The controller receives data from the continuous flow pump, and using a link, can be transmitted the data to the computer. 
Patients undergoing implantation of L-VAD, usually present with a previous history of sternotomy cardiac surgery and the positioning of a pacemaker / implantable cardioverter defibrillator (PM / ICD) (Figure 2 \& 3). These patients are subject to very long periods of hospitalization, because their condition must be stable and they must be able to handle the replacement of external batteries before being discharged to home. These patients have to undergo strict medical supervision and can access the cardiovascular rehabilitation programs; the results of functional recovery are very slow but verifiable [6,9-11]. The patient with L-VAD system is subject to a number of complications both during the hospital stay, and at home, in particular anemia, respiratory problems, recurrent infections in the insertion of the VAD cable, and on rare occasions, thrombosis [5-7]. In this study we evaluated the presence of osteopathic dysfunctions in patients with L-VAD, as we have verified that a text citing osteopathic issues is missing from the existing literature.

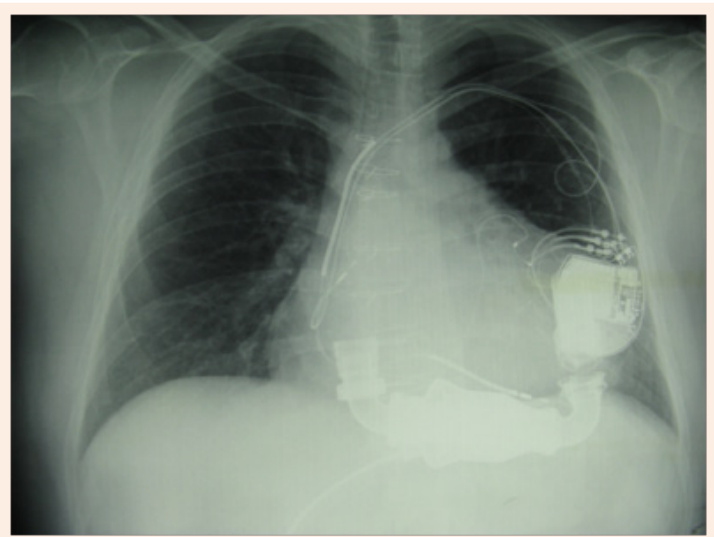

Figure 2: From the x-ray can be observed the pump, where to the left there is the tube which will arrive to the aorta, while on the right side the tube to the apex of the left ventricle. The patient also has a device of ICD-PM.

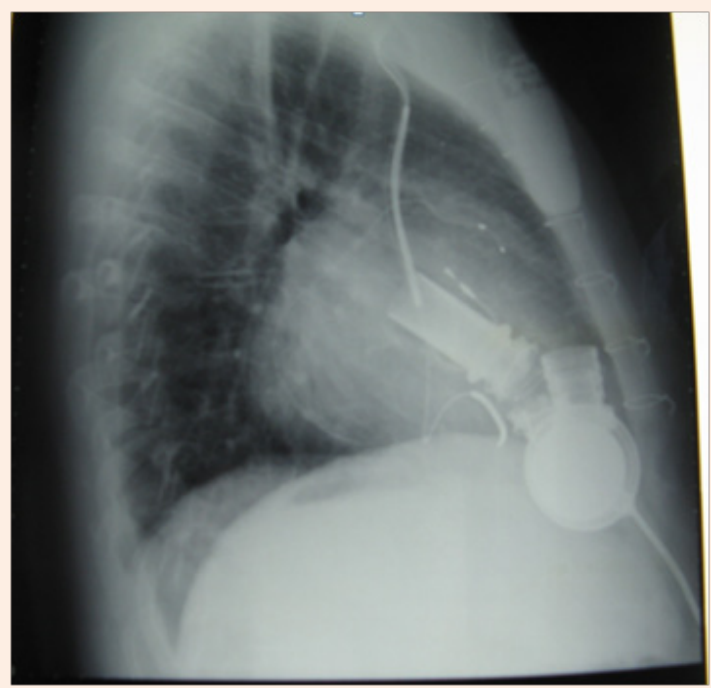

Figure 3: From the x-ray can be observed the pump above the diaphragm muscle, and the close connection with the heart.

\section{Methods}

\section{Setting and study partecipants}

In our centre, Don Carlo Gnocchi Foundation IRCCS S. Maria Nascente, during the time period between January 2011 and August 2016, 60 patients with L-VAD continuous flow implants were hospitalized, of which 55 were male and five women, with a minimum age of 23 years and a maximum age of 69 . The postintervention patients were from an inpatient hospital.

\section{Interventions}

During their hospitalization, they followed a cardiovascular rehabilitation program, and an osteopathic evaluation for the detection of possible faults, using an identical palpation approach for each patient. The osteopathic somatic dysfunction is defined as an "impaired or altered function of related components of the somatic (body framework) system: skeletal, arthrodial, and myofascial structures, and related vascular, lymphatic, and neural elements [12]. The diagnostic criterion for defining a somatic dysfunction must include abnormalities in tissue palpation, the asymmetry and the restriction of movement and the increase in muscular tone [12].1 The assessment was performed by the department on the second day after taking charge of the patient, before starting the cycle of cardiovascular rehabilitation. The patient was evaluated in supine and standing positions. The evaluation areas were chosen for ease of access, while ensuring the patient was comfortable.

All patients who underwent osteopathic evaluation displayed a respiratory diaphragm dysfunction, with a range of limited movement. With the patient in the supine position, palpation was performed by placing the hands under the diaphragm and at the same time, wrapping the fingers around the costal arches (Figure 4). Small lateral offsets were actively induced to evaluate the elasticity of tissues and whether there were preferential movement vectors. Evaluating the respiratory diaphragmatic area using active stimuli, the structure was listened to see if breathing had any cranial-caudal limitations, assessing the existence of possible restrictions.

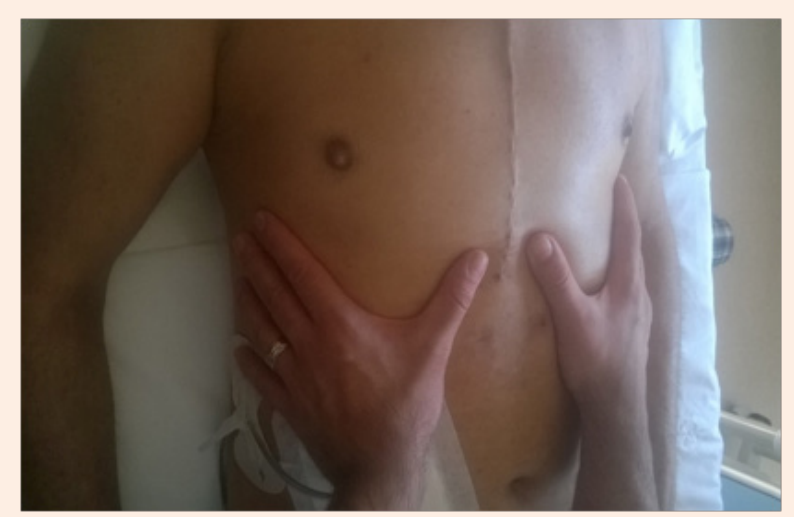

Figure 4: With the patient in the supine position, palpation was performed by placing the hands under the diaphragm and at the same time, wrapping the fingers around the costal arches. 
For the pelvic floor the operator positioned his hands bilaterally on the iliac bones, resting on the anterior-superior iliac spine. Perpendicular pressure was applied towards the bed, to understand the elastic response of the tissues and whether or not there were preferential movement vectors (Figure 5). Once the active areas are assessed, the structure was listened to in order to understand how diaphragmatic breathing is reflected on the pelvic area, assessing the existence of possible restrictions in the pelvic diaphragm area.

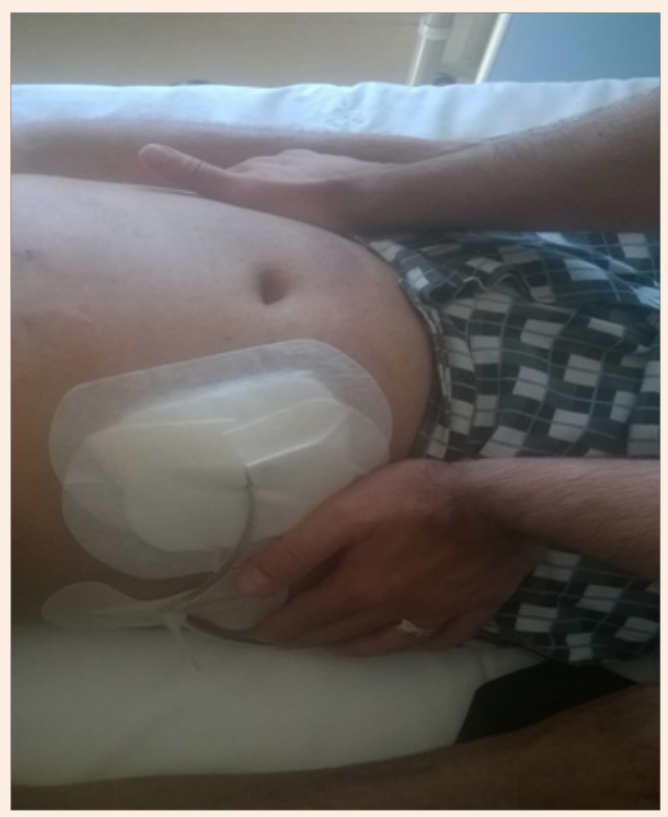

Figure 5: For the pelvic floor the operator positioned his hands bilaterally on the iliac bones, resting on the anterior-superior iliac spine. Perpendicular pressure was applied towards the bed, to understand the elastic response of the tissues and whether or not there were preferential movement vectors.

Applying light pressure to the cranial vector, the elasticity of the buccal floor was tested (Figure 6). In passive evaluation, always using the same palpatory approach, the operator assessed how the tissues behaved while the patient breathed.

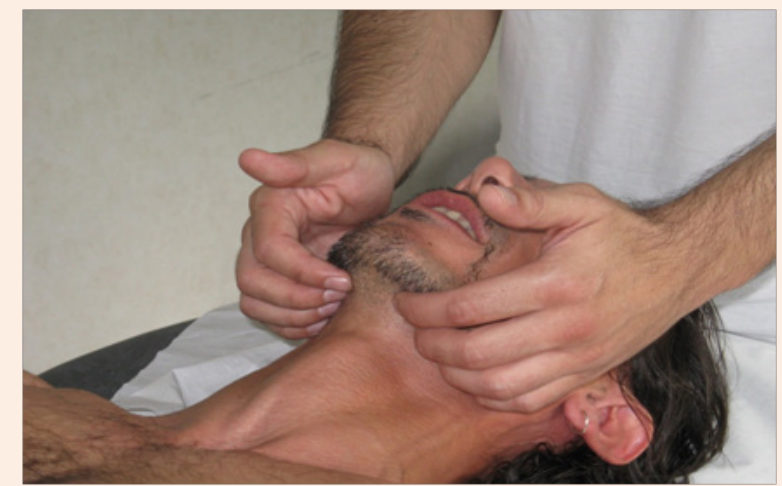

Figure 6: Place the fingertips in a medial position to the jawline and apply uniform pressure on both sides.
Palpating the paravertebral muscles as a whole, by placing the open palm on the muscles, had the effect of highlighting specific areas which stood out as having hypertonia and hypomobility of vertebral segments, when solicited to passive movement. Palpation was performed with open palm on the column, by pressing gently on the muscles, with the patient standing (Figure 7). The joint motion was evaluated with small passive movements; for the cervical spine, the patient was supine and the operator kept the cervical spine in the hands, soliciting with small lateral movements; the thoracic portion was assessed with the patient standing, with small trunk passive rotations and the operator's hand on each vertebral segment; the lumbar and sacral was evaluated with the patient supine, with one hand of the operator under the corresponding section and passive rotation of the legs with knees bent with the other hand (Figures 8-10). For the thoracic outlet area, with the patient in the supine position, the operator placed his hands on both sides so that the index and middle involved the clavicle (the bone between two fingers), the thumb was directed toward the C7-D1 level, and the remaining fingers on the area under the clavicular area.

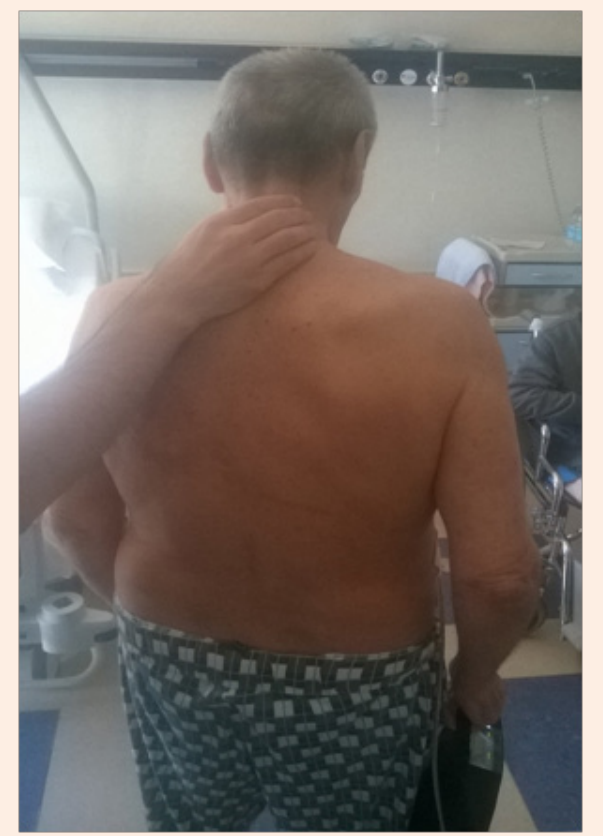

Figure 7: Palpation was performed with open palm on the column, by pressing gently on the muscles, with the patient standing.

General palpation of the craniosacral rhythm, by placing hands in open palm on the skull (little finger on the corner infero-lateral occiput, a middle finger on the asterion, the other middle finger on the tragus of the ear, the index on the pterion, and thumbs resting on the parietal bone), and later a hand palm open under the sacral bone with the patient supine.

\section{Outcome measurements}

The practitioner was instructed to report tissue abnormalities, restriction of movement in passive solicitation or listening, comparing the two sides of the body. The comprehensive 
palpation examination was carried out by more practitioners for each patient, without interchange evaluation.

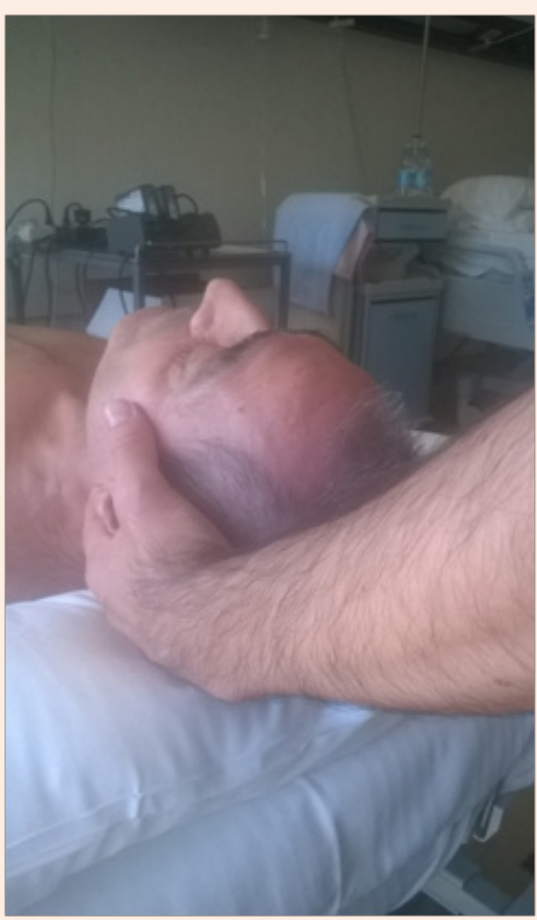

Figure 8: For the cervical spine, the patient was supine and the operator kept the cervical spine in the hands, soliciting with small lateral movements.

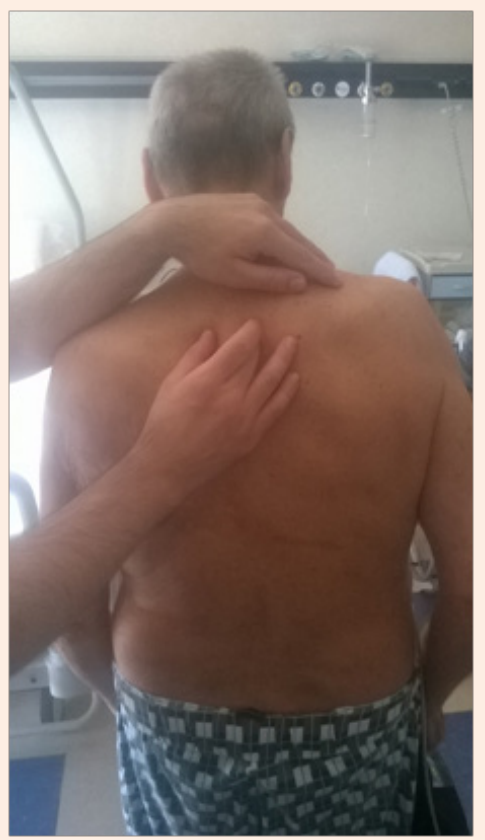

Figure 9: The thoracic portion was assessed with the patient standing, with small trunk passive rotations and the operator's hand on each vertebral segment.

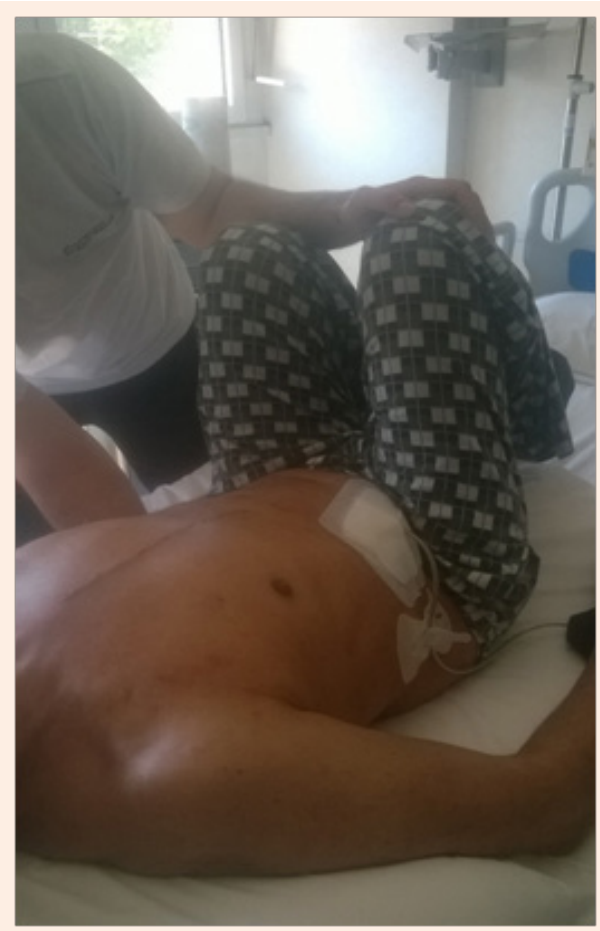

Figure 10: The lumbar and sacral was evaluated with the patient supine, with one hand of the operator under the corresponding section and passive rotation of the legs with knees bent with the other hand.

The research is observational. All patients were informed and allowed the osteopathic evaluation voluntarily. The patient was never in danger during the evaluation; all patients of our clinic are evaluated and are subjected to standard treatment, according to the Declaration of Helsinki - Ethical Principles for Medical Research Involving Human Subjects. Written informed consent was obtained from all patients.

\section{Results}

Of a total of 60 patients, 55 had a more pronounced left diaphragmatic motor dysfunction, while the remainder showed a major issue on the right. Osteopathic evaluation indicated that the side of the dome in diaphragmatic dysfunction is related to the hypertonia of the ipsilateral muscle of the pelvic floor. In palpating the floor of the mouth, and externally the medial branch of the mandible, we see the same phenomenon, that is to say an increase of tone that reflects the diaphragmatic side which is more in deficit.

The tone of the paravertebral muscles of the dorso-lumbar passage always appears to be in hypertonia, with reduced spinal mobility. All patients were found to have the same phenomenon of increased muscle tone and hypomobility of the facet joints at the level of C7-T4 and the occipital area sub-C1-C2. In 55 patients there was a level of C3-C4 hypertonia. Another dysfunction which always presents in all patients is thoracic outlet syndrome, with 
varying clinical intensity. Fifty five of the patients evaluated displayed paresthesia in the last two fingers of the hand with subjective intensity, especially the left little finger. The remaining number of patients reported the same disturbance, but to the right side. With the patient in the supine position, palpation for the thoracic outlet, highlighted an elevation of the first rib, a hypertonia of the subclavius muscle and of the pectoralis minor, in the side that had the disorder (Figures 11-13), (Table 1 \& 2).

Table 1: Somatic dysfunction.

\begin{tabular}{|c|c|c|c|c|}
\hline Patients & Diaphragm & Pelvic Floor & Floor of the Mouth & $\begin{array}{c}\text { Thoracic Outlet } \\
\text { Syndrome }\end{array}$ \\
\hline 55 & $\begin{array}{c}\text { Left somatic } \\
\text { dysfunction }\end{array}$ & $\begin{array}{c}\text { Left somatic } \\
\text { dysfunction }\end{array}$ & $\begin{array}{c}\text { Left somatic } \\
\text { dysfunction }\end{array}$ & Left Hypomobility C1-C2, \\
C3-C4, C7-T4 & Rigth \\
\hline 5 & $\begin{array}{c}\text { Right somatic } \\
\text { dysfunction }\end{array}$ & $\begin{array}{c}\text { Right somatic } \\
\text { dysfunction }\end{array}$ & $\begin{array}{c}\text { Hypomobility C1-C2, } \\
\text { C7-T4 } 4\end{array}$ \\
\hline
\end{tabular}

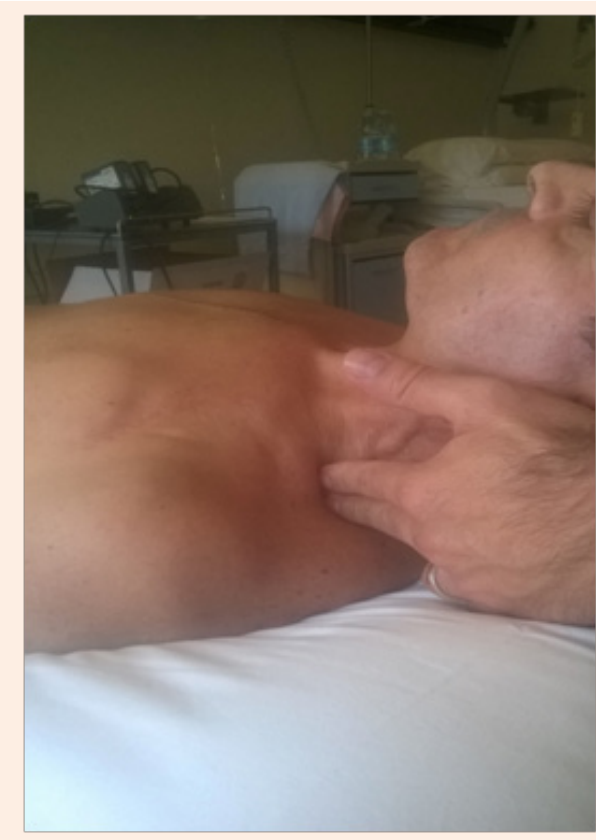

Figure 11: The operator evaluates the first rib, following with your fingers the posterior area of the neck, until the root of the neck; the examiner palpates the medial border of the first rib, which varies its position according to the breath.

Table 2: Graphic representing the major somatic dysfunction in 65 patients.

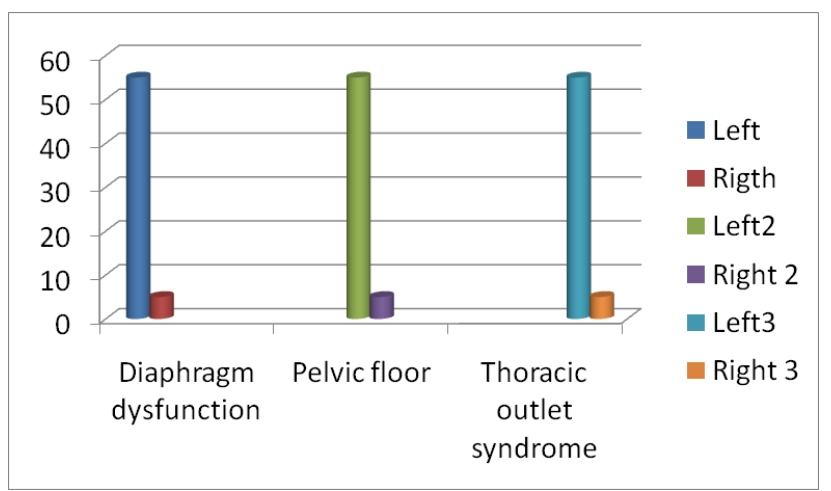

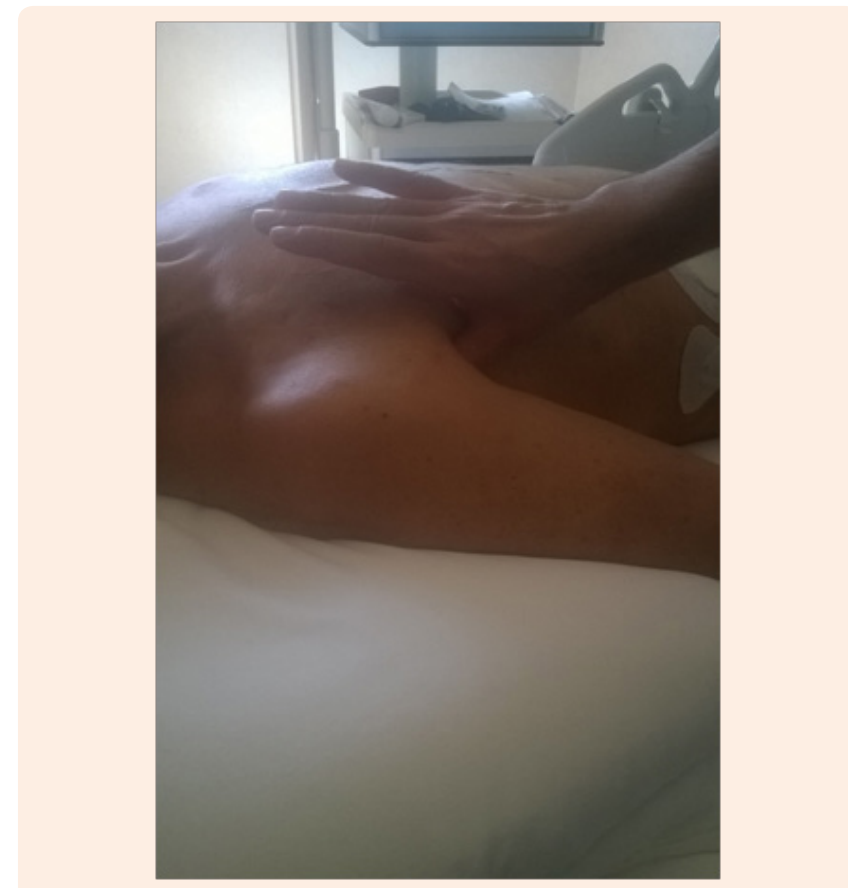

Figure 12: Under the axilla, the operator places the thumb with cranial direction towards the coracoid insertion of the muscle, with oblique vector.

General palpation of the craniosacral rhythm, displayed a slowing of the cranial rhythmic impulse in all patients tested (Figure 14).

\section{Discussion}

This manual evaluation is based on our experience comprising more than 20 years of clinical practice with patients affected by respiratory and cardiac diseases. The osteopathic dysfunctions that we found more frequently in patients undergoing implantation of L-VAD concerned an issue of diaphragmatic motor and a hypertonic muscles of the pelvic floor and the floor of the mouth, mirroring the side of the diaphragmatic dome dysfunction. The causes are many. People with CHF have an altered position of the diaphragm, in expiratory behaviour, with a non-physiological 
contractility rate [13]. Patient studies show a decrease in the strength of the diaphragm muscle and, in animal research, highlight a situation of myopathy with phenotypic changes of the contractile fibres $[14,15]$. People arriving at the point of L-VAD often have a previous history of sternotomy intervention. The surgical approach may injure the phrenic nerve, causing paralysis to the side of the diaphragmatic dome or greatly reducing its mobility. During the sternotomy procedure, the temperature drop causes a cooling lesion to the nerve, due to the close relationship between the phrenic and pericardium. This event is registered in greater proportion to the left nerve, in that the cooling is mainly

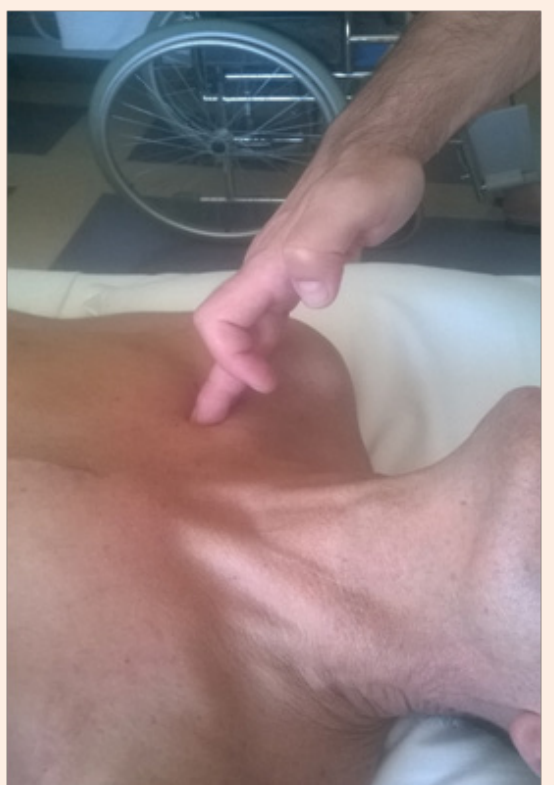

Figure 13: The operator puts the fingers below the clavicle, particularly in the portion of the middle third of the bone, experiencing a small strip of compact tissue.

The diaphragm muscle with its fascia and neurological relationships forms a network of afferent and efferent information that can affect the whole body. From a neurological point of view the contraction of the diaphragm muscle is preceded by the contraction of the floor of the mouth, and is synchronous with pelvic floor muscles [17]. The fascia connections to the diaphragm muscle, allows communication with the entire body network $[17,18]$. We can reasonably assume that a dysfunction of the diaphragm, will lead to functional alteration of the pelvic and buccal floors. The tone of the paravertebral muscles of the dorsolumbar area is always in hypertonia, with reduced passive spinal mobility, probably as a result of the presence of a diaphragmatic dysfunction. Our palpation evaluation highlighted hypertonia of the cervical muscle in 55 patients, at the level of $\mathrm{C} 3-\mathrm{C} 4$, resulting in hypomobility articulation. We hypothesize that this feedback is in relation to the suffering of the phrenic nerve, as a somatosomatic reflex [19]. In all patients, we noticed these phenomena at the level of the cervicodorsal track, in particular C7-T4, and the sub-occipital area, C1-C2. The change in muscle tone at cervicodorsal level could be a visceral-somatic reflex since several along the left side of the pericardium [16]. The nerve may suffer mechanical and ischemic damage by extracting the internal thoracic mammary artery, because of its proximity and is often used for bypass procedures [16]. Normally, we know from the literature that the functional electrophysiological recovery is recorded as occurring within 6-12 months of the procedure.16 However, there is no data on the restoration of electrical conductivity of the phrenic nerve in subjects who have undergone the procedure, in conjunction with chronic heart failure, or in patients with L-VAD.

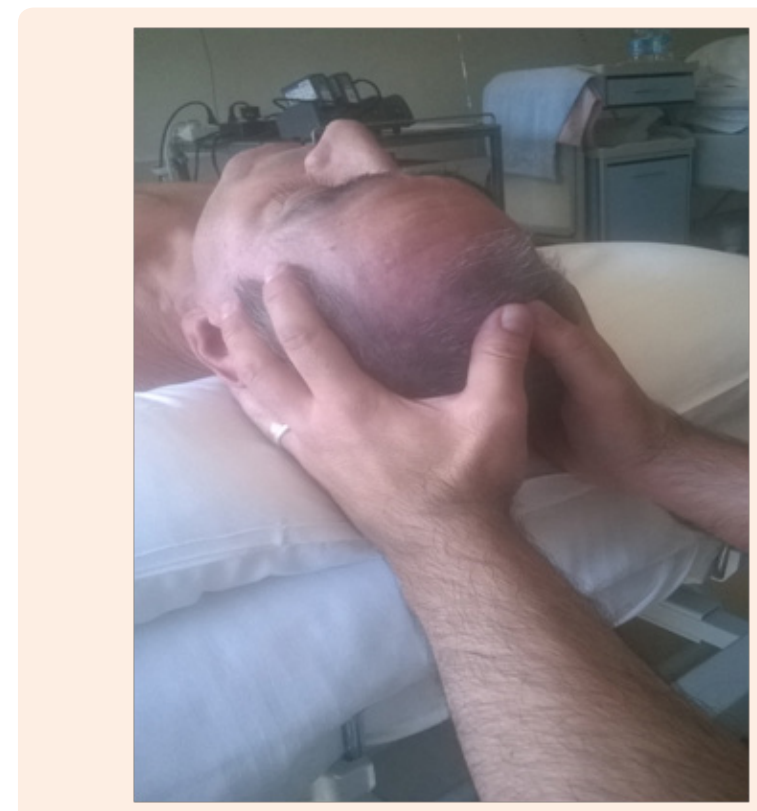

Figure 14: General palpation of the craniosacral rhythm, displayed a slowing of the cranial rhythmic impulse in all patients tested.

studies point out that the cell bodies of sympathetic afferent fibres are found in the dorsal root ganglia of C8-T9; these then run up the Lissauer track, progressing using the spinothalamic pathway [20]. Within this upward path somatic afferents can also be found [20]. Other studies with cardiovascular patients showed an osteopathic dysfunction in the spinal area.21 For the sub-occipital tract and the relationship between spinal C1 and C2, we noticed a strong muscle tension and hypomobility joint ratio. From the neurological point of view, electrophysiology studies involving the stimulation of cardiac nerves, suggest a close relationship between myocardial afferents, neck, jaw and spinal tract of C1C2 [20]. Other osteopathic research highlighted a dysfunction at the level of the cervical spine with high cardiovascular disease, probably caused by a visceral-somatic reflex via the afferent vagal nerve [21]. The vagal nerve has a close relationship with the aortic arch, particularly to the left [22]. We can also speculate as to the cause of such problems found, not only at the cervicodorsal area, in the area of $\mathrm{C} 1$ and $\mathrm{C} 2$ but also of the related muscles, is immobilization. These patients undergo a long period of bed rest, which can last for several months, before cardio 
surgical intervention to position the L-VAD, and also in the postsurgical phase. The posture adopted by the patient, to allow for comfortable bed rest with the neck and dorsal track elevated to a height of 45 degrees, is something which should be considered. This could cause structural and functional alterations of the cervical musculature. Another dysfunction that we detected in all patients, with varying degrees of clinical intensity, is the thoracic outlet syndrome. Palpation has highlighted a first rib in elevation and hypertonia of the subclavius and pectoralis minor muscle in the side in dysfunction. These dysfunctions lead to thoracic outlet syndrome, although we did not run specific surveys to diagnose the cause, such as a vascular or neurological compression [23,24]

The reasons for this finding are not well understood. This may be caused by a lesion induced posture, by the surgeon, on the patient, during the operation, as in other cardiac surgery patients without L-VAD the same symptoms can be found. General palpation of craniosacral rhythm showed a slowing of the cranial pulse. Taking into consideration that some studies have shown that the propagation of cardiovascular systolic waves and diaphragm muscle influence the craniosacral rhythm experienced by the osteopath during palpation, we can assume that this dysfunction is attributable to the current disease [25,26].25,26 According to a recent study, human touch can distinguish any slight variation measurable in microns [27]. We strongly believe that it is possible to train therapists with respect to appropriate palpation in order to check the mobility and function of the inspiratory muscle and to obtain additional clinical information on the therapeutic approach before and after physiotherapy [12].

It is important to remember that, as with other therapeutic techniques, whether manual or otherwise, conclusive scientific evidence is not available for every existing treatment; however this does not mean that the therapy is invalid, as otherwise there would be no new developments or improvement in rehabilitative treatments starategies [28]. In this regard, we wish to reiterate that evidence-based medicine (EBM), which originated in the second half of the $19^{\text {th }}$ century, is based on individual clinical expertise, best external evidence, patient values, and expectations: "External clinical evidence can inform, but can never replace, individual clinical expertise, and it is this expertise that decides whether the external evidence applies to the individual patient at all and, if so, how it should be integrated into a clinical decision [29]."

The limitations of the observational study are represented by the small number of practitioners and the inability to make comparisons with other working groups dealing with osteopathic patients with L-VAD.

\section{Conclusions}

The aim of the study was to investigate the presence and location of osteopathic dysfunctions in patients with L-VAD. Osteopathic dysfunctions that we found more frequently in patients undergoing implantation of L-VAD, address a problem and diaphragmatic hypertonia of the side corresponding to muscles of the pelvic and buccal floors. We detected hypertonia of the paraspinal muscles at the level of C1-C2, C3-C4 and C7$\mathrm{T} 4$, and highlighted that all patients complained of thoracic outlet syndrome; the causes of which remain speculative. The craniosacral rhythm is slow, probably as a result of the clinical and pathological history specific to the patient population evaluated.

\section{Disclosure}

The authors report no conflicts of interest in this work.

\section{References}

1. Fedele F, Gatto MC, D’Ambrosi A, Mancone M (2013) TNM-like classification: a new proposed method for heart failure staging. ScientificWorldJournal 2013: 175925.

2. Ahmed A, Allman RM, Fonarow GC, Love TE, Zannad F, et al. (2008) Incident heart failure hospitalization and subsequent mortality in chronic heart failure: a propensity-matched study. J Card Fail 14(3): 211-218.

3. Hirschl MM, Wollmann CG, Erhart F, Brunner W, Pfeffel F, et al (2013) Benefit of immediate beta-blocker therapy on mortality in patients with ST-segment elevation myocardial infarction. Crit Care Med 41(6): 1396-1404.

4. Brouwers C, Denollet J, de Jonge N, Caliskan K, Kealy J, et al. (2011) Patient-reported outcomes in left ventricular assist device therapy: a systematic review and recommendations for clinical research and practice. Circ Heart Fail 4(6):714-723.

5. Mallidi HR, Anand J, Cohn WE (2014) State of the art of mechanical circulatory support. Tex Heart Inst J 41(2): 115-120.

6. Chu SK, McCormick Z, Hwang S, Sliwa JA, Rydberg L (2014) Outcomes of Acute Inpatient Rehabilitation of Patients With Left Ventricular Assist Devices. PM R 6(11): 1008-1012.

7. Ginat D, Massey HT, Bhatt S, Dogra VS (2011) Imaging of mechanica cardiac assists devices. J Clin Imaging Sci 1: 21.

8. Yamakawa M, Kyo S, Yamakawa S, Ono M, Kinugawa K, et al. (2013) Destination therapy: the new gold standard treatment for heart failure patients with left ventricular assist devices. Gen Thorac Cardiovasc Surg 61(3): 111-117.

9. Compostella L, Russo N, Setzu T, Compostella C, Bellotto F (2014) Exercise performance of chronic heart failure patients in the early period of support by an axial-flow left ventricular assist device as destination therapy. Artif Organs 38(5): 366-373.

10. Alsara O, Reeves RK, Pyfferoen MD, Trenary TL, Engen DJ, et al (2014) Inpatient Rehabilitation Outcomes for Patients Receiving Left Ventricular Assist Device. Am J Phys Med Rehabil 93(10): 860868.

11. Hayes K, Leet AS, Bradley SJ, Holland AE (2012) Effects of exercise training on exercise capacity and quality of life in patients with a left ventricular assist device: a preliminary randomized controlled trial. J Heart Lung Transplant 31(7): 729-734.

12. Chila AG (2010) Foundations for Osteopathic Medicine. ( $3^{\text {rd }}$ edn), Lippincott Williams, USA.

13. Caruana L, Petrie MC, McMurray JJ, MacFarlane NG (2001) Altered diaphragm position and function in patients with chronic heart failure. Eur J Heart Fail 3(2): 183-187.

14. Hughes PD, Polkey MI, Harrus ML, Coats AJ, Moxham J, et al. (1999) Diaphragm strength in chronic heart failure. Am J Respir Crit Care Med 160(2): 529-534. 
15. Lima AR, Martinez PF, Damatto RL, Cezar MD, Guizoni DM, et al (2014) Heart failure-induced diaphragm myopathy. Cell Physiol Biochem 34(2): 333-345.

16. Aguirre VJ, Sinha P, Zimmet A, Lee GA, Kwa L, et al. (2013) Phrenic nerve injury during cardiac surgery: mechanisms, management and prevention. Heart Lung Circ 22(11): 895-902.

17. Bordoni B, Zanier E (2013) Anatomic connections of the diaphragm influence of respiration on the body system. J Multidiscip Healthc 6: 281-291.

18. Bordoni B, Zanier E (2011) Skin, fascias, and scars: symptoms and systemic connections. J Multidiscip Healthc 7: 11-24.

19. Johnston WL, Golden WJ (2001) Segmental definition--Part IV Updating the differential for somatic and visceral inputs. J Am Osteopath Assoc 101(5): 278-283.

20. Rosen SD (2012) From heart to brain: the genesis and processing of cardiac pain. Can J Cardiol 28(2 Suppl): S7-S19.

21. Wieting JM, Beal C, Roth GL, Gorbis S, Dillard L, et al. (2013) The effect of osteopathic manipulative treatment on postoperative medical and functional recovery of coronary artery bypass graft patients. J Am Osteopath Assoc 113(5): 384-393.
22. Akhter SA (2011) The heart and pericardium. Thorac Surg Clin 21(2): 205-217.

23. Singh VK, Jeyaseelan L, Kyriacou S, Ghosh S, Sinisi M, et al. (2014) Diagnostic value of magnetic resonance imaging in thoracic outlet syndrome. J Orthop Surg (Hong Kong) 22(2): 228-231.

24. Freischlag J, Orion K (2014) Understanding thoracic outlet syndrome. Scientifica (Cairo) 2014: 248163.

25. Nelson KE, Sergueef N, Glonek T (2006) Recording the rate of the cranial rhythmic impulse. J Am Osteopath Assoc 106(6): 337-341.

26. Whedon JM, Glassey D (2009) Cerebrospinal fluid stasis and its clinical significance. Altern Ther Health Med 15(3): 54-60.

27. Skedung L, Arvidsson M, Chung JY, Stafford CM, Berglund B, et al. (2013) Feeling small: exploring the tactile perception limits. Sci Rep 3: 2617.

28. Bordoni B, Zanier E (2015) The continuity of the body: hypothesis of treatment of the five diaphragms. J Altern Complement Med 21(4): 237-242.

29. Masic I, Miokovic M, Muhamedagic B (2008) Evidence based medicine - new approaches and challenges. Acta Inform Med 16(4): 219-225. 